\title{
Locally anisotropic cosmological systems in modified two measure and massive gravity theories
}

\author{
Sergiu I. Vacaru \\ Rector's Department, Alexandru Ioan Cuza University, UAIC \\ Al. Lapuşneanu str., nr. 14, UAIC - Corpus R, office 323; Iaşi, Romania, 700057; and* \\ M. Planck Inst. Physics (W. Heisenberg Inst.), Föhringer Ring 6, München D-80805, Germany; \\ Leibniz Univ. Hannover, Instit. Theoretical Physics, Appelstrasse 2, Hannover 30167, Germany \\ E-mails: sergiu.vacaru@uaic.ro; Sergiu.Vacaru@gmail.com
}

\begin{abstract}
We outline a geometric method of constructing generic off-diagonal and diagonal cosmological solutions of effective Einstein equations modeling modified gravity theories with two non-Riemannian volume forms and associated bimetric and/or biconnection geometric structures. Such solutions are determined by generating functions, effective sources and integration constants and characterized by nonholonomic frame torsion effects. In the physical Einstein frame, the constructions involve: (i) nonlinear re-parametrization symmetries of the generating functions and effective sources; (ii) effective potentials for the scalar field with possible two flat regions which allows unified description of locally anisotropic and/or isotropic early universe inflation related to acceleration cosmology and dark energy; (iii) there are "emergent universes" described by off-diagonal and diagonal solutions for certain nonholonomic phases and parametric cosmological evolution resulting in various inflationary phases; (iv) we can reproduce in two measure theories massive gravity effects.
\end{abstract}

Keywords: Modified and massive gravity; two measure theories; bi-metrics and biconnections; effective Einstein gravity; off-diagonal cosmological solutions; nonholonomic dynamical Weyl-scale symmetry breaking; (anisotropic) inflation, dark energy; reconstructing procedure.

Modern cosmology has a very important task to provide a theoretical description of many aspects of observable universe with exponential expansion (inflation), particle creation, and radiation, acceleration and dark energy / matter effects. The physical community almost accepted the idea that the Einstein gravity and standard particle physics have to be modified in order to elaborate self-consistent quantum gravity theories and describe existing experimental and observational data in modern cosmology. In result, a number of modified gravity theories, MGTs, and cosmological scenarios have been elaborated in the last 15 years. In this paper, we summarize our recent results on geometric methods in constructing exact inhomogeneous cosmological solutions in MGTs ${ }^{1-6}$.

MGTs can be constructed as geometric models using two independent nonRiemannian volume-forms ${ }^{1} \Phi(A)$ and ${ }^{2} \Phi(B)$ as in two measure theories, TMTs, which can be related to be-metric, $\widehat{\mathbf{g}}_{\alpha \beta} \mathbf{g}_{\alpha \beta}$, and be-connection theories, and more general functional for modification, of type $\epsilon \mathbf{f}(\widetilde{\mathbf{R}})$, than $\epsilon R^{2}$ if $\widehat{\mathbf{D}} \rightarrow \nabla$. For instance, the Lagrange density functional ${ }^{f, \mu} \mathcal{L}=\mathbf{F}(\widetilde{\mathbf{R}})$ is determined similar to a modified

*DAAD fellowship affiliations for two host institutions 
massive gravity by a mass-deformed scalar curvature ${ }^{\text {a }}$

$$
\tilde{\mathbf{R}}:=\widehat{\mathbf{R}}+2 \mu^{2}\left(3-\operatorname{tr} \sqrt{\mathbf{g}^{-1} \mathbf{q}}-\operatorname{det} \sqrt{\mathbf{g}^{-1} \mathbf{q}}\right),
$$

where $\mu$ is the graviton's mass and $\mathbf{q}=\left\{\mathbf{q}_{\alpha \beta}\right\}$ is the so-called non-dynamical reference metric.

Applying such a calculus to actions on generalized non-Riemannian spaces, ${ }^{F, \mu} \mathcal{S}+{ }^{m} \mathcal{S}$, where ${ }^{m} \mathcal{L}$ is the Lagrangian for matter fields, with ${ }^{1} \mathbf{F}(\tilde{\mathbf{R}}):=$ $d \mathbf{F}(\tilde{\mathbf{R}}) / d \tilde{\mathbf{R}}$, see details in ${ }^{5-8}$, we obtain the modified gravitational field equations

$$
\widehat{\mathbf{R}}_{\mu \nu}={ }^{F, \mu} \widehat{\boldsymbol{\Upsilon}}_{\mu \nu}
$$

where ${ }^{F, \mu} \widehat{\boldsymbol{\Upsilon}}_{\mu \nu}={ }^{m} \widehat{\boldsymbol{\Upsilon}}_{\mu \nu}+{ }^{f} \widehat{\boldsymbol{\Upsilon}}_{\mu \nu}+{ }^{\mu} \widehat{\boldsymbol{\Upsilon}}_{\mu \nu}$, for

$$
\begin{aligned}
{ }^{m} \widehat{\boldsymbol{\Upsilon}}_{\mu \nu}= & \frac{1}{2 M_{P}^{2}}{ }^{m} \widehat{\mathbf{T}}_{\alpha \beta},{ }^{f} \widehat{\boldsymbol{\Upsilon}}_{\mu \nu}=\left(\frac{\mathbf{F}}{2^{1} \mathbf{F}}-\frac{\widehat{\mathbf{D}}^{2}{ }^{1} \mathbf{F}}{{ }^{1} \mathbf{F}}\right) \widehat{\mathbf{g}}_{\mu \nu}+\frac{\widehat{\mathbf{D}}_{\mu} \widehat{\mathbf{D}}_{\nu}{ }^{1} \mathbf{F}}{{ }^{1} \mathbf{F}}, \\
{ }^{\mu} \widehat{\boldsymbol{\Upsilon}}_{\mu \nu}= & \left.-2 \mu^{2}\left[\left(3-\operatorname{tr} \sqrt{\widehat{\mathbf{g}}^{-1} \mathbf{q}}-\operatorname{det} \sqrt{\widehat{\mathbf{g}}^{-1} \mathbf{q}}\right)-\frac{1}{2} \operatorname{det} \sqrt{\widehat{\mathrm{g}}^{-1} \mathbf{q}}\right)\right] \widehat{\mathbf{g}}_{\mu \nu} \\
& +\frac{\mu^{2}}{2}\left\{\mathbf{q}_{\mu \rho}\left[\left(\sqrt{\widehat{\mathrm{g}}^{-1} \mathbf{q}}\right)^{-1}\right]_{\nu}^{\rho}+\mathbf{q}_{\nu \rho}\left[\left(\sqrt{\widehat{\mathbf{g}}^{-1} \mathbf{q}}\right)^{-1}\right]^{\rho}{ }_{\mu}\right\} .
\end{aligned}
$$

The field equations for massive gravity (1) are constructed as nonholonomic biconnection deformations of the Einstein equations for the Levi Civita connection when the source $\widehat{\Upsilon}_{\beta \gamma} \rightarrow{ }^{F, \mu} \widehat{\Upsilon}_{\mu \nu}$, with generalized sources (2).

We can integrate in very general forms the effective Einstein equations considering bi-metric structures

$$
\begin{aligned}
& \widehat{\mathbf{g}}_{\alpha \beta}=\Theta \mathbf{g}_{\alpha \beta}, \text { for } \Theta={ }^{1} \chi-{ }^{2} \chi \epsilon{ }^{1} \mathbf{f}\left({ }^{1} L+{ }^{1} M, \mu\right) \\
& { }^{e f} L=\Theta^{-1}\left\{{ }^{1} L+{ }^{1} M+{ }^{2} \chi \Theta^{-1}\left[{ }^{2} L+{ }^{1} M+\epsilon{ }^{1} \mathbf{f}\left({ }^{1} L+{ }^{1} M, \mu\right)\right]\right\} .
\end{aligned}
$$

It is possible to generate in explicit form integral varieties of systems of PDEs of type (1) via frame and coordinate transforms and parameterizations with $\mathrm{r}$ a time like coordinate $y^{4}=t\left(i^{\prime}, i, k, k^{\prime}, \ldots=1,2\right.$ and $\left.a, a^{\prime}, b, b^{\prime}, \ldots=3,4\right)$, when

$$
\begin{aligned}
\widehat{\mathbf{g}} & =\widehat{\mathrm{g}}_{\alpha^{\prime} \beta^{\prime}} \mathbf{e}^{\alpha^{\prime}} \otimes \mathbf{e}^{\beta^{\prime}}=g_{i}\left(x^{k}\right) d x^{i} \otimes d x^{j}+\omega^{2}\left(x^{k}, y^{3}, t\right) h_{a}\left(x^{k}, t\right) \mathbf{e}^{a} \otimes \mathbf{e}^{a}, \\
\mathbf{e}^{3} & =d y^{3}+n_{i}\left(x^{k}, t\right) d x^{i}, \mathbf{e}^{4}=d t+w_{i}\left(x^{k}, t\right) d x^{i},
\end{aligned}
$$

for nontrivial

$$
\begin{aligned}
\left\{g_{i^{\prime} j^{\prime}}\right\} & =\operatorname{diag}\left[g_{i}\right], g_{1}=g_{2}=e^{\psi\left(x^{k}\right)} ;\left\{g_{a^{\prime} b^{\prime}}\right\}=\operatorname{diag}\left[h_{a}\right], h_{a}=h_{a}\left(x^{k}, t\right) ; \\
N_{i}^{3} & =n_{i}\left(x^{k}, t\right) ; N_{i}^{4}=w_{i}\left(x^{k}, t\right) ;
\end{aligned}
$$

and $\widehat{\Upsilon}_{\alpha^{\prime} \beta^{\prime}}=\operatorname{diag}\left[\Upsilon_{i} ; \Upsilon_{a}\right]$,

$$
\begin{array}{r}
\text { for } \Upsilon_{1}=\Upsilon_{2}=\widetilde{\Upsilon}\left(x^{k}\right)={ }^{e f} \widetilde{\Upsilon}\left(x^{k}\right)+{ }^{m} \widetilde{\Upsilon}\left(x^{k}\right)+{ }^{f} \widetilde{\Upsilon}\left(x^{k}\right)+{ }^{\mu} \widetilde{\Upsilon}\left(x^{k}\right), \\
\Upsilon_{3}=\Upsilon_{4}=\Upsilon\left(x^{k}, t\right)={ }^{e f} \Upsilon\left(x^{k}, t\right)+{ }^{m} \Upsilon\left(x^{k}, t\right)+{ }^{f} \Upsilon\left(x^{k}, t\right)+{ }^{\mu} \Upsilon\left(x^{k}, t\right) .
\end{array}
$$

\footnotetext{
aThere are various ambiguities and controversies in different approaches to massive gravity when modifications by mass terms are postulated for different Lagrange densities; in this paper, we consider a "toy model" when terms of type $\mathbf{f}(\widetilde{\mathbf{R}}, \mu)$ and/or $\mathbf{f}(\mathbf{R})+\mu \ldots$ can me modeled by the same MGT but for different classes of nonholonomic constraints and different classes of solutions.
} 
We use parameterizations $g_{1}=g_{2}=e^{\psi\left(x^{i}\right)}$ and $h_{a}\left(x^{k}, t\right)$ for $i, j, \ldots=1,2$ and $a, b, \ldots=3,4$; and $\mathrm{N}$-connection coefficients $\mathbf{N}_{i}^{3}=n_{i}\left(x^{k}, t\right)$ and $\mathbf{N}_{i}^{4}=w_{i}\left(x^{k}, t\right)$. Introducing brief denotations for partial derivatives like $a^{\bullet}=\partial_{1} a, b^{\prime}=\partial_{2} b, h^{*}=$ $\partial_{4} h=\partial_{t} h$ and defining the values

$$
\alpha_{i}=h_{3}^{*} \partial_{i} \varpi, \beta=h_{3}^{*} \varpi^{*}, \gamma=\left(\ln \left|h_{3}\right|^{3 / 2} /\left|h_{4}\right|\right)^{*}
$$

for a generating function $\varpi:=\ln \mid h_{3}^{*} / \sqrt{\left|h_{3} h_{4}\right|}$, we shall also use the value $\Psi:=e^{\varpi}$.

Using ansatz (3), we transform (1) into a nonlinear system of PDEs with decoupling property for $\psi\left(x^{i}\right), h_{a}\left(x^{k}, t\right), w_{i}\left(x^{k}, t\right), n_{i}\left(x^{k}, t\right)$ (un-known functions),

$$
\psi^{\bullet \bullet}+\psi^{\prime \prime}=2 \widetilde{\Upsilon}, \varpi^{*} h_{3}^{*}=2 h_{3} h_{4} \Upsilon, n_{i}^{* *}+\gamma n_{i}^{*}=0, \beta w_{i}-\alpha_{i}=0
$$

This system can be solved in very general forms with dependence on generating, $\Psi$, and integration functions and posses another very important property which allows us to re-define the generating function,

$$
\Psi \longleftrightarrow \widetilde{\Psi}, \text { when } \Lambda\left(\Psi^{2}\right)^{*}=|\Upsilon|\left(\widetilde{\Psi}^{2}\right)^{*}
$$

and $\Lambda \Psi^{2}=\widetilde{\Psi}^{2}|\Upsilon|+\int d t \widetilde{\Psi}^{2}|\Upsilon|^{*}$, for $\widetilde{\Psi}:=\exp \widetilde{\varpi}$ and any prescribed values of effective (for different types of contributions $e f, m, f, \mu$ ) cosmological constants in $\Lambda={ }^{e f} \Lambda+{ }^{m} \Lambda+{ }^{f} \Lambda+{ }^{\mu} \Lambda$ associated respectively to

$$
\Upsilon\left(x^{k}, t\right)={ }^{e f} \Upsilon\left(x^{k}, t\right)+{ }^{m} \Upsilon\left(x^{k}, t\right)+{ }^{f} \Upsilon\left(x^{k}, t\right)+{ }^{\mu} \Upsilon\left(x^{k}, t\right)
$$

and effective source

$$
\Xi:=\int d t \Upsilon\left(\widetilde{\Psi}^{2}\right)^{*}={ }^{e f} \Xi+{ }^{m} \Xi+{ }^{f} \Xi+{ }^{\mu} \Xi
$$

when ${ }^{e f} \Xi:=\int d t^{e f} \Upsilon\left(\widetilde{\Psi}^{2}\right)^{*},{ }^{m} \Xi:=\int d t^{m} \Upsilon\left(\widetilde{\Psi}^{2}\right)^{*},{ }^{f} \Xi:=\int d t{ }^{f} \Upsilon\left(\widetilde{\Psi}^{2}\right)^{*}$.

Different types of inhomogeneous cosmological solutions are determined by corresponding classes of and effective sources

$$
\begin{array}{ll}
\text { generating functions: } & \psi\left(x^{k}\right), \widetilde{\Psi}\left(x^{k}, t\right), \omega\left(x^{k}, y^{3}, t\right) \\
\text { effective sources: } & \widetilde{\Upsilon}\left(x^{k}\right) ;{ }^{e f} \Xi\left(x^{k}, t\right),{ }^{m} \Xi\left(x^{k}, t\right),{ }^{f} \Xi\left(x^{k}, t\right),{ }^{\mu} \Xi\left(x^{k}, t\right), \\
& \text { or }{ }^{e f} \Upsilon\left(x^{k}, t\right),{ }^{m} \Upsilon\left(x^{k}, t\right),{ }^{f} \Upsilon\left(x^{k}, t\right),{ }^{\mu} \Upsilon\left(x^{k}, t\right) \\
\text { integration: } & \text { integration cosmological constants: }{ }^{e f} \Lambda,{ }^{m} \Lambda,{ }^{f} \Lambda,{ }^{\mu} \Lambda \\
\text { integration functions: } & { }_{1} n_{i}\left(x^{k}\right) \text { and }{ }_{2} n_{i}\left(x^{k}\right)
\end{array}
$$

We can generate solutions with any nontrivial ${ }^{e f} \Lambda,{ }^{m} \Lambda,{ }^{f} \Lambda,{ }^{\mu} \Lambda$ even any, or all, effective source ${ }^{e f} \Upsilon,{ }^{m} \Upsilon,{ }^{f} \Upsilon,{ }^{\mu} \Upsilon$ can be zero. 
Example: Massive gravity modifications of flat regions. We can integrate in generic off-diagonal form such TMT systems as subclasses of solutions, when

$$
\begin{aligned}
d s^{2}= & \bar{a}^{2}\left(x^{k}, t\right)\left[\eta_{1}\left(x^{k}, t\right)\left(d x^{1}\right)^{2}+\eta_{2}\left(x^{k}, t\right)\left(d x^{2}\right)^{2}\right] \\
& +\bar{a}^{2}\left(x^{k}, t\right) \widehat{h}_{3}\left(x^{k}, t\right)\left[d y^{3}+\left({ }_{1} n_{i}+{ }_{2} n_{i} \int d t \frac{\left(\widetilde{\Psi}^{*}\right)^{2}}{\widetilde{\Psi}^{3}(\text { ef } \Xi+\mu \Xi)}\right) d x^{i}\right]^{2} \\
& -\left[d t+\frac{\partial_{i}\left(\text { ef } \Xi+\mu{ }^{\mu} \Xi\right)}{\left(e^{e f} \Xi+\mu \Xi\right)^{*}} d x^{i}\right]^{2},
\end{aligned}
$$

for $\quad{ }^{e f} \Xi:=\int d t{ }^{\text {ef }} \Upsilon\left(\widetilde{\Psi}^{2}\right)^{*}, \quad{ }^{\mu} \Xi:=\int d t{ }^{\mu} \Upsilon\left(\widetilde{\Psi}^{2}\right)^{*}$. We write $\widetilde{\Psi} \rightarrow \bar{\Psi}$, when the generating function is chosen to satisfy the conditions

$$
\bar{a}^{2} \widehat{h}_{3}=\omega^{2} h_{3}=\frac{h_{3}}{\left|h_{4}\right|}=\frac{\bar{\Psi}^{2} \mid{ }^{e f} \Xi+\mu}{4\left({ }^{e \mu} \Lambda\right)\left(\bar{\Psi}^{*}\right)^{2}} .
$$

In general, such nonhomogeneous locally anisotropic configurations are with nontrivial nonholonomically induced canonical d-torsion which can be constrained to be zero for corresponding subclasses of generating functions and sources.

We study off-cosmological solutions depending only on time like coordinate when $\widetilde{a}\left(x^{k}, t\right) \simeq \widetilde{a}(t)$ and $\widetilde{\Psi}\left(x^{k}, t\right) \simeq \widetilde{\Psi}(t)$ and the generation function $\widetilde{\Psi}(t)$. The formula relating variations of ${ }^{e \mu} \Upsilon(t)$ to the variation of the second auxiliary 3-index antisymmetric d-tensor field $\mathbf{B}_{\alpha \beta \gamma}$ in ${ }^{2} \Phi(\mathbf{B})$, a particular case is given by

$$
{ }^{2} \Phi(\overline{\mathbf{B}}) / \sqrt{|\widehat{\mathbf{g}}|}={ }^{2} \bar{\chi}={ }^{2} \tilde{\chi}+{ }^{\mu} \chi=\text { const },
$$

where the constant ${ }^{\mu} \chi$ is zero for $\mu=0$ and $\left|{ }^{\mu} \chi\right| \ll\left|{ }^{2} \tilde{\chi}\right|$. Another assumption is that we can formulate a TMT theory corresponding to "pure" $\mu$-deformations of GR even $\epsilon=0$. Such formulas have to be generalized for nontrivial $\mu$, when

$$
{ }^{1} \mathbf{f}\left({ }^{1} L+{ }^{1} M+{ }^{\mu} M, \mu\right)=\left.\frac{d \mathbf{f}(\widehat{\mathbf{R}}, \mu)}{d \widehat{\mathbf{R}}}\right|_{\widehat{\mathbf{R}}={ }^{1} L+{ }^{1} M} \rightarrow{ }^{1} U-{ }^{1} M-{ }^{\mu} M
$$

is a version of generalized Starobinsky relation and approximations of type $\tilde{\mathbf{R}} \simeq$ $\widehat{\mathbf{R}}+\widetilde{\mu}^{2}$.

The resulting formulas for effective potential contain additional $\mu$-terms

$$
\begin{aligned}
{ }^{e} U & =\frac{\left({ }^{1} U-{ }^{1} M-{ }^{\mu} M\right)^{2}}{4^{2} \widetilde{\chi}\left[{ }^{2} U+{ }^{2} M+\left({ }^{1} U-{ }^{1} M-{ }^{\mu} M\right)^{2}\right]} \\
& \simeq{ }^{e} \bar{U}=\left\{\begin{array}{cc}
{ }^{[-]} \bar{U}=\frac{\left({ }^{1} a\right)^{2}}{4\left({ }^{2} \tilde{\chi}+{ }^{\mu} \chi\right)\left[{ }^{2} a+\epsilon\left({ }^{1} a\right)^{2}\right]} & \text { for } \varphi \rightarrow-\infty \\
{[+] \bar{U}=\frac{\left({ }^{1} M+{ }^{\mu} M\right)^{2}}{4\left({ }^{2} \tilde{\chi}+{ }^{\mu} \chi\right)\left[{ }^{2} M+\epsilon\left({ }^{1} M\right)^{2}\right]}} & \text { for } \varphi \rightarrow+\infty
\end{array} \mid .\right.
\end{aligned}
$$

The $A$ - and $B$-functions can also contain contributions of $\mu$-terms,

$$
\begin{aligned}
& \bar{A} \simeq\left\{\begin{array}{c}
{[-] \bar{A}=\frac{{ }^{2} a+\frac{1}{2}{ }^{2} b^{1} a}{{ }^{2} a+\epsilon\left({ }^{1} a\right)^{2}}} \\
{[+] \widetilde{A}=\frac{{ }^{2} M}{{ }^{2} M+\epsilon\left({ }^{1} M+{ }^{\mu} M\right)^{2}}}
\end{array} \mid\right. \\
& \text { and } \bar{B} \simeq\left\{\begin{array}{c}
{[-] \bar{B}=-\left({ }^{2} \widetilde{\chi}+{ }^{\mu} \chi\right) \frac{{ }^{2} b / 4-\epsilon\left({ }^{2} a+{ }^{2} b{ }^{1} a\right)}{{ }^{2} a+\epsilon\left({ }^{1} a\right)^{2}}} \\
{[+] \bar{B}=\epsilon\left({ }^{2} \widetilde{\chi}+{ }^{\mu} \chi\right) \frac{{ }^{\mu} M}{{ }^{2} M+\epsilon\left({ }^{1} M+{ }^{\mu} M\right)^{2}}}
\end{array} \mid,\right.
\end{aligned}
$$


when ${ }^{[-]} \bar{A}$ is not modified. We conclude that solutions with nontrivial generating functions for nontrivial massive gravity terms modelled as effective TMT theories may also describe non-singular "emergent universes" with corresponding modifications.

\section{Acknowledgments}

$\mathrm{SV}$ is supported by a travel grant from MG14 and reports certain research related to his basic activity at UAIC, a DAAD fellowship and the Program IDEI, PN-II-IDPCE-2011-3-0256. He is grateful for DAAD hosting to D. Lüst and O. Lechtenfeld.

\section{References}

1. S. Vacaru, Phys. Lett. B 690, 224-228 (2010).

2. S. Vacaru, Int. J. Mod. Phys. D 21, 1250072 (2012).

3. P. Stavrinos, S. Vacaru, Class. Quant. Grav. 30, 055012 (2013).

4. S. Vacaru, Eur. Phys. J. C 74, 3132 (2014).

5. S. Vacaru, Eur. Phys. J. C 75, 176 (2015).

6. E. Elizalde, S. Vacaru, Gen. Relat. Grav. 47, 64 (2015).

7. E. Elizalde, S. Vacaru, Eur. Phys. J. P 130, 119 (2015).

8. S. Vacaru, Phys. Lett. B 752, 27 (2016). 